\title{
The Lagrange Multiplier Method for Dirichlet's Problem
}

\author{
By James H. Bramble
}

\begin{abstract}
The Lagrange multiplier method of Babuška for the approximate solution of Dirichlet's problem for second order elliptic equations is reformulated. Based on this formulation, new estimates for the error in the solution and the boundary flux are given. Efficient methods for the solution of the approximate problem are discussed.
\end{abstract}

1. Introduction. The purpose of this paper is to consider a method introduced by Babuška [3] for approximating the solution of Dirichlet's problem for second order elliptic operators. An important aspect of this method is that the approximate solution involves only natural boundary conditions. The point of view here is similar to that taken by Falk [7] in that a family of solutions $u(\sigma)$ of the differential equation is introduced, where $\sigma$ is a function defined on the boundary. In fact $\sigma=\partial u / \partial \nu+\alpha u$. For each such function $\sigma, u(\sigma)$ satisfies the given differential equation, and we seek to determine $\sigma$ so that $u(\sigma)$ takes on specified boundary values. By approximating the space in which $\sigma$ lies and that in which $u$ lies we are led to a method of approximation which is a reformulation of Babuška's method. The advantage of this formulation is that we obtain new optimal error estimates for the error in $u$ in norms weaker than the energy norm. We also obtain new estimates for the boundary flux approximation. It is finally shown how the approximation $\sigma_{k}$ to $\sigma$ may be obtained numerically as a limit of a rapidly convergent sequence of solutions to problems with natural boundary conditions. This is done by introducing a "discrete surface Laplacian" with the help of which we may formulate our equations in terms of a well-conditioned matrix problem which may be solved efficiently by the conjugate gradient method.

An outline of the paper is as follows. In Section 2 we introduce the Dirichlet problem and its decomposition into solutions of natural boundary value problems. Relevant a priori estimates are there given. Section 3 concerns the spaces of approximating functions in our domain $\Omega$ and Section 4 introduces spaces of approximating functions on the boundary $\partial \Omega$. Section 5 contains a reformulation of Babuška's method and there the a priori estimates which are the basis for the error estimates and computational methods are proved. The error estimates are proved in Section 6, and finally in Section 7 the "discrete surface Laplacian" is introduced and the estimates of Section 5 are used to develop efficient computational procedures.

Received January 1, 1981.

1980 Mathematics Subject Classification. Primary 65N30.

(c) 1981 American Mathematical Society 0025-5718/81/0000-0100/\$03.75 
2. Notation and Preliminaries. We shall be concerned with the solution $u$ of the problem

$$
\begin{aligned}
L u=f & \text { in } \Omega, \\
u=g & \text { on } \partial \Omega,
\end{aligned}
$$

where $\Omega$ is a bounded domain in $d$-dimensional space $R^{d}$ with smooth boundary $\partial \Omega$. The operator $L$ is given formally by

$$
L u=-\sum_{i, j=1}^{d} \frac{\partial}{\partial x_{i}}\left(a_{i j} \frac{\partial u}{\partial x_{j}}\right)+a u,
$$

where $a_{i j}$ is a uniformly positive definite matrix of smooth functions in $\Omega$ and $a>0$ is also smooth. We shall not consider the exact amount of smoothness necessary but will assume that the solution $u \in H^{s}(\Omega)$ provided $f \in H^{s-2}(\Omega)$ and $g \in$ $H^{s-1 / 2}(\partial \Omega)$. Here $H^{s}(\Omega)$ and $H^{s}(\partial \Omega)$ are the usual Sobolev spaces of functions defined on $\Omega$ and $\partial \Omega$, respectively, with $s$ a positive real number, and $H^{s}(\Omega)$ and $H^{s}(\partial \Omega)$ are the respective duals of $H^{-s}(\Omega)$ and $H^{-s}(\partial \Omega)$ for $s<0$. The norms in $H^{s}(\Omega)$ and $H^{s}(\partial \Omega)$ will be denoted by $\|\cdot\|_{s}$ and $|\cdot|_{s}$, respectively.

The above statement is expressed here by the well-known a priori estimate (cf. [9])

$$
\|u\|_{s} \leqslant C\left(\|L u\|_{s-2}+|u|_{s-1 / 2}\right) .
$$

For the purpose of this paper we wish to consider the boundary value problems with natural boundary conditions

$$
\begin{array}{ll}
L v=0 & \text { in } \Omega, \\
\alpha v+\frac{\partial v}{\partial \nu}=\sigma & \text { on } \partial \Omega,
\end{array}
$$

and

$$
\begin{array}{ll}
L w=f & \text { in } \Omega, \\
\alpha w+\frac{\partial w}{\partial \nu}=0 & \text { on } \partial \Omega .
\end{array}
$$

Here $\alpha \geqslant 0$ is a constant chosen so that $a+\alpha>0$ and $\partial / \partial \nu$ is the outward conormal derivative on $\partial \Omega$ relative to $L$. The introduction of $\alpha$ avoids the appearance of semidefinite forms or the requirement that $a>0$ in order that (2.3) and (2.4) have unique solutions. Let $\langle\phi, \psi\rangle$ be the $\mathscr{L}_{2}$ inner product on the boundary and also the pairing between $H^{s}(\partial \Omega)$ and $H^{-s}(\partial \Omega)$, and let $(\phi, \psi)$ be the $\varrho_{2}$ inner product on $\Omega$ and also the pairing between $H^{s}(\Omega)$ and $H^{-s}(\Omega)$. We shall denote the generalized Dirichlet integral by $A_{\alpha}(\cdot, \cdot)$; it is given by

$$
A_{\alpha}(\phi, \psi)=\sum_{i, j=1}^{d} \int_{\Omega} a_{i j} \frac{\partial \phi}{\partial x_{i}} \frac{\partial \psi}{\partial x_{j}} d x+(a \phi, \psi)+\alpha\langle\phi, \psi\rangle .
$$

It is defined on $H^{1}(\Omega) \times H^{1}(\Omega)$ and is positive definite.

In this notation we may formulate $(2.3)$ as follows: $v \in H^{1}(\Omega)$ satisfies

$$
A_{\alpha}(v, \chi)=\langle\sigma, \chi\rangle
$$

for all $\chi \in H^{1}(\Omega)$. Set $v=G \sigma$. It is well known (cf. [9]) that $G: H^{s}(\partial \Omega) \rightarrow$ $H^{s+3 / 2}(\Omega)$ as a bounded operator; i.e. for $\theta \in H^{s}(\partial \Omega)$

$$
\|G \theta\|_{s+3 / 2}<C|\theta|_{s}
$$


Here and in the sequel $C$ denotes a generic constant not necessarily the same in any two places. At times also $C_{0}$ and $C_{1}$ will denote generic constants.

We observe finally that since $G$ maps $\varrho_{2}(\partial \Omega)$ into $H^{1}(\Omega)$, the trace of $G \theta$ on $\partial \Omega$ is in $H^{1 / 2}(\partial \Omega)$. Hence we may consider $G: \digamma_{2}(\partial \Omega) \rightarrow \digamma_{2}(\partial \Omega)$, and it follows easily from the definition that $G$ is selfadjoint.

We have the following lemmas concerning $G$.

LEMMA 2.1. There exist positive constants $C_{0}$ and $C_{1}$ such that

$$
C_{0}|\theta|_{-1 / 2}^{2}<\langle G \theta, \theta\rangle \leqslant C_{1}|\theta|_{-1 / 2}^{2}
$$

for $\theta \in H^{-1 / 2}(\partial \Omega)$.

Proof. Note that $\langle G \theta, \theta\rangle=A_{\alpha}(G \theta, G \theta)<C\|G \theta\|_{1}^{2}$. The second inequality now follows from (2.5). The first inequality follows by a simple duality argument. Let $L \psi=0$ and consider

$$
\langle\theta, \psi\rangle=A_{\alpha}(G \theta, \psi)<C A_{\alpha}^{1 / 2}(G \theta, G \theta)\|\psi\|_{1} .
$$

By (2.2) it follows that

$$
\frac{\langle\theta, \psi\rangle}{|\psi|_{1 / 2}}\left\langle C A_{\alpha}^{1 / 2}(G \theta, G \theta)=C\langle G \theta, \theta\rangle^{1 / 2}\right.
$$

But $\psi \in H^{1 / 2}(\partial \Omega)$ may be arbitrary and hence

$$
|\theta|_{-1 / 2}=\sup _{\psi \in H^{1 / 2}(\partial \Omega)} \frac{\langle\theta, \psi\rangle}{|\psi|_{1 / 2}}\left\langle C\langle G \theta, \theta\rangle^{1 / 2} .\right.
$$

In a similar fashion we may prove the following

LEMMA 2.2. Let $s$ be a real number. Then there exist constants $C_{0}$ and $C_{1}$ such that

$$
C_{0}|\theta|_{s}<|G \theta|_{s+1}<C_{1}|\theta|_{s}
$$

for all $\theta \in H^{s}(\partial \Omega)$.

Proof. The first inequality for $s>0$ is just an application of a trace theorem (cf. [8]) and (2.2). We have

$$
|\theta|_{s}=\left|\alpha G \theta+\frac{\partial G \theta}{\partial \nu}\right|_{s} \leqslant C\left(|G \theta|_{s}+\|G \theta\|_{s+3 / 2}\right)<C|G \theta|_{s+1} .
$$

When $s=-\frac{1}{2}$ it follows from Lemma 2.1. By interpolation it is true for any $s>-\frac{1}{2}$. Its validity for $s<-\frac{1}{2}$ is proved by a simple duality argument.

The second inequality for $s>-\frac{1}{2}$ follows from a trace inequality and (2.5). Again a duality argument proves it for $s<-\frac{1}{2}$.

In a similar manner we set the solution $w$ of (2.4) to be $T f$. Again it is well known that $T: H^{s}(\Omega) \rightarrow H^{s+2}(\Omega)$ as a bounded operator. Now with this notation we shall represent the solution $u$ of $(2.1)$ as

$$
u=G \sigma+T f .
$$

With $f \in H^{s-2}(\Omega)$ and $\sigma \in H^{s-3 / 2}(\partial \Omega), u \in H^{s}(\Omega)$. Conversely, given $f \in$ $H^{s-2}(\Omega)$ and $g \in H^{s-1 / 2}(\partial \Omega)$ there exists a unique $\sigma \in H^{s-3 / 2}(\partial \Omega)$ such that

$$
G \sigma=g-T f \text { on } \partial \Omega,
$$

and then $u$ is given by (2.8). In fact $\sigma=\alpha u+\partial u / \partial \nu$. It is unique by Lemma 2.2. 
It will be from this point of view that we will approximate $u$; i.e. we will approximate $G, T$ and $\sigma$ to obtain an approximation to $u$.

3. Approximating Spaces on $\Omega$. For $0<h<1$ let $\left\{S_{h}\right\}$ be a family of finite dimensional subspaces of $H^{1}(\Omega)$. Let $r \geqslant 2$ be an integer. We shall assume that for $\phi \in H^{l}(\Omega)$ with $1 \leqslant l \leqslant r$,

$$
\inf _{\chi \in S_{h}}\|\phi-\chi\|_{j} \leqslant C h^{l-j}\|\phi\|_{l}, \quad j<1 .
$$

We now define the operators $G_{h}: H^{-1 / 2}(\partial \Omega) \rightarrow S_{h}$ and $T_{h}: H^{-1}(\Omega) \rightarrow S_{h}$ by

$$
A_{\alpha}\left(G_{h} \theta, \chi\right)=\langle\theta, \chi\rangle, \quad \forall \chi \in S_{h},
$$

and

$$
A_{\alpha}\left(T_{h} f, \chi\right)=(f, \chi), \quad \forall \chi \in S_{h} .
$$

These are just the so-called standard Ritz-Galerkin approximations to $G$ and $T$. We recall some well-known properties. Let $P_{1}$ be the orthogonal projection onto $S_{h}$ with respect to the norm (equivalent to the $H^{1}(\Omega)$-norm) induced by $A_{\alpha}$; i.e.

$$
A_{\alpha}\left(\phi-P_{1} \phi, \chi\right)=0, \quad \forall \chi \in S_{h} .
$$

Then with this notation we see that

$$
G_{h} \sigma=P_{1} G \sigma \text { and } T_{h} f=P_{1} T f,
$$

so that we have immediately from the approximation assumption and standard duality arguments the following well-known results (cf. [2], [4]).

LEMMA 3.1. There exists a constant $C$ such that

$$
\left|\left(G-G_{h}\right) \sigma\right|_{j-1 / 2}+\left\|\left(G-G_{h}\right) \sigma\right\|_{j} \leqslant C h^{l-j}\|G \sigma\|_{l}<C h^{l-j}\|u\|_{l}
$$

and

$$
\left|\left(T-T_{h}\right) f\right|_{j-1 / 2}+\left\|\left(T-T_{h}\right) f\right\|_{j} \leqslant C h^{l-j}\|T f\|_{l}<C h^{l-j}\|u\|_{l}
$$

for $2-r \leqslant j \leqslant 1 \leqslant l \leqslant r, \sigma \in H^{l-3 / 2}(\partial \Omega), f \in H^{l-2}(\Omega)$ and $u=G \sigma+T f$.

Note that the restriction to $\Omega$ of continuous piecewise polynomials of degree $r-1$ on a quasi-uniform triangulation of $R^{2}$ or a rectangular mesh of "width" $h$ are examples of spaces $S_{h}$ satisfying (3.1).

4. Approximating Spaces on $\partial \Omega$. For $0<k<1$ let $\left\{\dot{S}_{k}\right\}$ be a family of finite dimensional subspaces of $H^{n}(\partial \Omega), n \geqslant 0$. Let $\dot{r} \geqslant 1$ be an integer. We shall suppose that for $\phi \in H^{l}(\partial \Omega)$ with $j \leqslant n, j \leqslant l \leqslant \dot{r}$,

$$
\inf _{x \in \dot{S}_{k}}|\phi-\chi|_{j} \leqslant C k^{l-j}|\phi|_{l} .
$$

We further assume that for $j \leqslant m \leqslant n$

$$
|\phi|_{m} \leqslant C k^{j-m}|\phi|_{j}
$$

for all $\phi \in \dot{S}_{k}$.

The conditions (4.1) and (4.2) together imply that for any given $j_{0}<n$ there is an operator $\pi_{k}: H^{j_{0}}(\partial \Omega) \rightarrow \dot{S}_{k}$ with

$$
\left|\phi-\pi_{k} \phi\right|_{j} \leqslant C_{j_{0}} k^{l-j}|\phi|_{l}
$$

uniformly in $j$ and $l$ with $j_{0} \leqslant j \leqslant l \leqslant \dot{r}, j \leqslant n$. This result may be found in [5]. 
Finally we denote by $p_{0}$ the $\mathscr{L}_{2}(\partial \Omega)$ orthogonal projection onto $\dot{S}_{k}$; i.e. for $v \in$ $\digamma_{2}(\partial \Omega) \equiv H^{0}(\partial \Omega)$

$$
\left\langle p_{0} v, \theta\right\rangle=\langle v, \theta\rangle, \quad \forall \theta \in \dot{S}_{k} .
$$

By a standard duality argument we have

LEMMA 4.1. Let $\dot{S}_{k} \subset H^{n}(\partial \Omega)$. Then

$$
\left|\left(I-p_{0}\right) \phi\right|_{j}<C k^{l-j}|\phi|_{l}
$$

for $\phi \in H^{l}(\partial \Omega)$, with $-n \leqslant l \leqslant \dot{r}$ and $-\dot{r} \leqslant j \leqslant \min (l, n)$.

We shall also need the following lemma concerning the boundedness of $p_{0}$ in other norms.

LemMa 4.2. Suppose $\dot{S}_{k} \subset H^{n}(\partial \Omega)$. Then

$$
\left|p_{0} \phi\right|_{s}<C|\phi|_{s}
$$

for $|s| \leqslant \min (n, \dot{r})$.

The proof follows from Lemma 4.1.

We note here that the operator $p_{0} G_{h}: \dot{S}_{k} \rightarrow \dot{S}_{k}$ is selfadjoint; i.e. for $\phi, \psi \in \dot{S}_{k}$,

$$
\left\langle p_{0} G_{h} \phi, \psi\right\rangle=\left\langle G_{h} \phi, \psi\right\rangle=A_{\alpha}\left(G_{h} \phi, G_{h} \psi\right)=\left\langle\phi, p_{0} G_{h} \psi\right\rangle \text {. }
$$

This operator will play a central role in what follows.

5. Babuška's Method Reformulated. As noted in Section 2 we may write $u$ as in (2.8) and (2.9).

The approximate problem motivated in this way is: Find $\sigma_{k} \in \dot{S}_{k}$ such that

$$
p_{0} G_{h} \sigma_{k}=p_{0}\left(g-T_{h} f\right) \text {. }
$$

The approximation to $u$ is defined as

$$
u_{h k}=G_{h} \sigma_{k}+T_{h} f
$$

From the definitions of $G_{h}$ and $T_{h}$ it follows that

$$
A_{\alpha}\left(u_{h k}, \chi\right)=\left\langle\sigma_{k}, \chi\right\rangle+(f, \chi), \quad \forall \chi \in S_{h},
$$

and

$$
\left\langle u_{h k}-g, \theta\right\rangle=0, \quad \forall \theta \in \dot{S}_{k} .
$$

These equations are the same as those of Babuška [3].

The first estimate which we shall give is an analogue of Lemma 2.1. A condition relating $h$ to $k$ will be required.

LEMMA 5.1. For $h<\varepsilon k$ with $\varepsilon$ sufficiently small there exist positive constants $C_{0}$ and $C_{1}$ such that

$$
C_{0}|\theta|_{-1 / 2}^{2}<\left\langle G_{h} \theta, \theta\right\rangle\left\langle C_{1}|\theta|_{-1 / 2}^{2}\right.
$$

for all $\theta \in \dot{S}_{k}$.

Proof. By Lemma 2.1,

$$
C_{0}|\theta|_{-1 / 2}^{2}\left\langle\left\langle G_{h} \theta, \theta\right\rangle+\left\langle\left(G-G_{h}\right) \theta, \theta\right\rangle\left\langle C_{1}|\theta|_{-1 / 2}^{2}\right.\right.
$$


Hence it suffices to show, for $\theta \in \dot{S}_{k}$, that $\left|\left\langle\left(G-G_{h}\right) \theta, \theta\right\rangle\right|<\delta|\theta|_{-1 / 2}^{2}$ where $\delta$ is small with $\varepsilon$. By definition

$$
\begin{aligned}
\left\langle\left(G-G_{h}\right) \theta, \theta\right\rangle & =A_{\alpha}\left(\left(G-G_{h}\right) \theta, G \theta\right)=A_{\alpha}\left(\left(G-G_{h}\right) \theta,\left(G-G_{h}\right) \theta\right) \\
& <C\left\|\left(G-G_{h}\right) \theta\right\|_{1}^{2}<C h^{2 \beta}\|G \theta\|_{1+\beta}^{2}
\end{aligned}
$$

for $0<\beta<\frac{1}{2}$, where we have used Lemma 3.1.

By (2.5)

$$
\|G \theta\|_{1+\beta}<C|\theta|_{\beta-1 / 2} \text {. }
$$

By assumption (4.2) with $s=\beta-\frac{1}{2}$ and $j=-\frac{1}{2}$

$$
|\theta|_{\beta-1 / 2}<C k^{-\beta}|\theta|_{-1 / 2} \text {. }
$$

Hence combining (5.4)-(5.6) we see that

$$
\left|\left\langle\left(G-G_{h}\right) \theta, \theta\right\rangle\right|<C(h / k)^{2 \beta}|\theta|_{-1 / 2}^{2}<C \varepsilon^{2 \beta}|\theta|_{-1 / 2}^{2} .
$$

The result follows for $\varepsilon$ small enough.

Lemma 5.1 clearly implies the existence and uniqueness of $\sigma_{k}$ since $p_{0} G_{h} \theta=0$ for $\theta \in \dot{S}_{k}$ implies that $\theta=0$.

Another stability estimate will be needed in our derivation of the error estimates and the computational method.

LEMMA 5.2. Suppose that $h<\varepsilon k$ with $\varepsilon$ sufficiently small. Set $m=\min (r-3 / 2, \dot{r})$ and let $-m \leqslant s+1 \leqslant 1$. Assume that $\dot{S}_{k} \subset \mathscr{L}_{2}(\partial \Omega)$ and furthermore that $\dot{S}_{k} \subset$ $H^{1}(\partial \Omega)$ if $0<s+1<1$. Then there exist positive constants $C_{0}$ and $C_{1}$ such that

$$
C_{0}|\theta|_{s}<\left|p_{0} G_{h} \theta\right|_{s+1}<C_{1}|\theta|_{s}
$$

for all $\theta \in \dot{S}_{k}$.

Proof. We shall consider the cases $-m<s+1<0$ and $s=0$. The result will then follow by interpolation.

Consider first $s$ with $-m<s+1<0$. Using Lemma 2.2 and the triangle inequality, we see that

$$
C_{0}|\theta|_{s} \leqslant\left|\left(G-G_{h}\right) \theta\right|_{s+1}+\left|\left(I-p_{0}\right) G_{h} \theta\right|_{s+1}+\left|p_{0} G_{h} \theta\right|_{s+1}
$$

and

$$
\left|p_{0} G_{h} \theta\right|_{s+1} \leqslant\left|\left(G-G_{h}\right) \theta\right|_{s+1}+\left|\left(I-p_{0}\right) G_{h} \theta\right|_{s+1}+C_{1}|\theta|_{s} .
$$

Hence it suffices to prove that

$$
\left|\left(G-G_{h}\right) \theta\right|_{s+1} \leqslant C \varepsilon|\theta|_{s}, \quad \frac{3}{2}-r<s+1<0,
$$

and

$$
\left|\left(I-p_{0}\right) G_{h} \theta\right|_{s+1}^{2}<C\left|p_{0} G_{h} \theta\right|_{s+1}|\theta|_{s}, \quad-\dot{r}<s+1<0,
$$

for $\theta \in \dot{S}_{k}$.

To prove (5.7) we use Lemma 3.1 with $j-\frac{1}{2}=s+1$ and $l=\frac{3}{2}$ to obtain

$$
\left|\left(G-G_{h}\right) \theta\right|_{s+1} \leqslant C h^{-s}\|G \theta\|_{3 / 2} \text {. }
$$

From (2.5), (4.2) and the fact that $-s>1$ we see that

$$
h^{-s}\|G \theta\|_{3 / 2}<C h^{-s}|\theta|<C(h / k)^{-s}|\theta|_{s}<C \varepsilon|\theta|_{s}
$$

which, together with (5.9), proves (5.7). 
To prove (5.8) we apply Lemma 4.1 to obtain

$$
\left|\left(I-p_{0}\right) G_{h} \theta\right|_{s+1}^{2}<C k^{-2 s-1}\left|G_{h} \theta\right|_{1 / 2}^{2}
$$

By the trace theorem and the definition of $G_{h}$

$$
\left|G_{h} \theta\right|_{1 / 2}^{2}\left\langleC \| G _ { h } \theta \| _ { 1 } ^ { 2 } \left\langle C A_{\alpha}\left(G_{h} \theta, G_{h} \theta\right)=C\left\langle\theta, p_{0} G_{h} \theta\right\rangle .\right.\right.
$$

Hence, using (4.2),

$$
\left|\left(I-p_{0}\right) G_{h} \theta\right|_{s+1}^{2}<C k^{-s-1}\left|p_{0} G_{h} \theta\right| k^{-s}|\theta|<C\left|p_{0} G_{h} \theta\right|_{s+1}|\theta|_{s}
$$

which is (5.9), and hence the lemma is proved when $-m<s+1<0$.

It remains to consider $0<s+1<1$ when $\dot{S}_{k} \subset H^{1}(\partial \Omega)$. We only look at the case $s=0$. The full result will follow by interpolation. Let $\psi \in \dot{S}_{k}$ be the unique solution of $p_{0} G_{h} \psi=\theta$ which exists and satisfies $|\psi|_{-1}<C|\theta|$ by virtue of the first part of the proof. Hence

$$
|\theta|^{2}=\left\langle\theta, p_{0} G_{h} \psi\right\rangle=\left\langle p_{0} G_{h} \theta, \psi\right\rangle\left\langle C\left|p_{0} G_{h} \theta\right|_{1}|\theta|,\right.
$$

so that $|\theta| \leqslant C\left|p_{0} G_{h} \theta\right|_{1}$. Finally, since $\dot{S}_{k} \subset H^{1}(\partial \Omega)$,

$$
\left|p_{0} G_{h} \theta\right|_{1}<\left|p_{0}\left(G-G_{h}\right) \theta\right|_{1}+\left|p_{0} G \theta\right|_{1}<\left|p_{0}\left(G-G_{h}\right) \theta\right|_{1}+C|G \theta|_{1}
$$

by Lemma 4.2. Using (4.2), Lemma 3.1, (2.5) and (2.7), we see that

$$
\begin{aligned}
\left|p_{0} G_{h} \theta\right|_{1} & <C k^{-1}\left|\left(G-G_{h}\right) \theta\right|+C|G \theta|_{1} \\
& <C(h / k)\|G \theta\|_{3 / 2}+C|G \theta|_{1}<C|G \theta|_{1}<C|\theta|
\end{aligned}
$$

which completes the proof of the lemma.

6. Error Estimates. The following theorems are the main error estimates. The first is the energy norm estimate given by Babuška [3]. The estimates in weaker norms are sharper than those of [3]. These in turn give rise to an accurate method for calculating the flux on the boundary.

THEOREM 1. Let $r<\dot{r}+\frac{3}{2}$ and $h<\varepsilon k$ for $\varepsilon$ small enough for Lemmas 5.1 and 5.2 to hold. Then

$$
\left|\sigma-\sigma_{k}\right|_{-1 / 2}+\left\|u-u_{h k}\right\|_{1}<C\left(h^{r-1}\|u\|_{r}+k^{\dot{r}+1 / 2}|\sigma|_{\dot{r}}\right)
$$

Proof. We note that

$$
u-u_{h k}=\left(G-G_{h}\right) \sigma+\left(T-T_{h}\right) f+G_{h}\left(\sigma-\sigma_{k}\right) .
$$

Hence to estimate $\left\|u-u_{h k}\right\|_{1}$, by Lemma 3.1 it suffices only to estimate $G_{h}\left(\sigma-\sigma_{k}\right)$. But since $G_{h}=P_{1} G$, we have that

$$
\left\|G_{h}\left(\sigma-\sigma_{k}\right)\right\|_{1}<C\left\|G\left(\sigma-\sigma_{k}\right)\right\|_{1}<C\left|\sigma-\sigma_{k}\right|_{-1 / 2}
$$

by (2.5). Thus we have reduced the estimate to that of $\left|\sigma-\sigma_{k}\right|_{-1 / 2}$. By (4.3)

$$
\begin{aligned}
\left|\sigma-\sigma_{k}\right|_{-1 / 2} & <C\left(\left|\left(I-\pi_{k}\right) \sigma\right|_{-1 / 2}+\left|\pi_{k} \sigma-\sigma_{k}\right|_{-1 / 2}\right) \\
& <C\left(k^{\dot{r}+1 / 2}|\sigma|_{\dot{r}}+\left|\pi_{k} \sigma-\sigma_{k}\right|_{-1 / 2}\right) .
\end{aligned}
$$

By Lemma 5.1

$$
\left|\pi_{k} \sigma-\sigma_{k}\right|_{-1 / 2}<C\left\langle G_{h}\left(\pi_{k} \sigma-\sigma_{k}\right), \pi_{k} \sigma-\sigma_{k}\right\rangle
$$


Using the boundary conditions (5.1) and (2.9)

$$
p_{0} G_{h}\left(\pi_{k} \sigma-\sigma_{k}\right)=p_{0}\left[G_{h}\left(\pi_{k} \sigma-\sigma\right)+\left(G_{h}-G\right) \sigma+\left(T_{h}-T\right) f\right],
$$

we see from (6.1) that

$$
\left|\pi_{k} \sigma-\sigma_{k}\right|_{-1 / 2} \leqslant C\left(\left|\left(G_{h}-G\right) \sigma\right|_{1 / 2}+\left|\left(T_{h}-T\right) f\right|_{1 / 2}+\left|G_{h}\left(\pi_{k} \sigma-\sigma\right)\right|_{1 / 2}\right) .
$$

Hence applying Lemma 3.1 and a trace inequality, we obtain

$$
\left|\pi_{k} \sigma-\sigma_{k}\right|_{-1 / 2} \leqslant C\left(h^{r-1}\|u\|_{r}+\left\|G_{h}\left(\pi_{k} \sigma-\sigma\right)\right\|_{1}\right) .
$$

But

$$
\left\|G_{h}\left(\pi_{k} \sigma-\sigma\right)\right\|_{1} \leqslant C\left\|G\left(\pi_{k} \sigma-\sigma\right)\right\|_{1} \leqslant C\left|\pi_{k} \sigma-\sigma\right|_{-1 / 2}
$$

by the stability of $P_{1}$ and (2.5). Collecting these results and using (4.3) completes the proof.

THEOREM 2. Let $\dot{S}_{k} \subset H^{n}(\partial \Omega)$ with $0 \leqslant n<r-\frac{3}{2}, r<\dot{r}+\frac{3}{2}$ and $h<\varepsilon k$ as in Theorem 1. Then for $0 \leqslant i \leqslant r-2$,

$$
\left\|u-u_{h k}\right\|_{-i} \leqslant\left\{\begin{array}{l}
C\left(h^{r+i}\|u\|_{r}+k^{\dot{r}+3 / 2+i}|\sigma|_{r}\right), \quad 0<i<n-\frac{1}{2}, \\
C\left((k / h)^{i+1 / 2-n} h^{r+i}\|u\|_{r}+k^{\dot{r}+3 / 2+i}|\sigma|_{r}\right), \quad n-\frac{1}{2}<i<r-2 .
\end{array}\right.
$$

Proof. As noted in the proof of Theorem 1 it suffices to estimate $G_{h}\left(\sigma-\sigma_{k}\right)$. By the triangle inequality and Lemma 3.1

$$
\left\|G_{h}\left(\sigma-\sigma_{k}\right)\right\|_{-i} \leqslant\left\|G\left(\sigma-\sigma_{k}\right)\right\|_{-i}+h^{1+i}\left\|G\left(\sigma-\sigma_{k}\right)\right\|_{1} .
$$

Now by (2.5) with $s=-\frac{1}{2}$ and Theorem 1 it remains only to estimate $\left\|G\left(\sigma-\sigma_{k}\right)\right\|_{-i}$ for $0 \leqslant i \leqslant r-2$. By (2.5) and (4.3)

$$
\begin{aligned}
\left\|G\left(\sigma-\sigma_{k}\right)\right\|_{-i} & \leqslant C\left|\sigma-\sigma_{k}\right|_{-i-3 / 2}<C\left(\left|\left(I-\pi_{k}\right) \sigma\right|_{-i-3 / 2}+\left|\pi_{k} \sigma-\sigma_{k}\right|_{-i-3 / 2}\right) \\
& \leqslant C\left(k^{\dot{r}+3 / 2+i}|\sigma|_{\dot{r}}+\left|\pi_{k} \sigma-\sigma_{k}\right|_{-i-3 / 2}\right) .
\end{aligned}
$$

It remains to estimate $\left|\pi_{k} \sigma-\sigma_{k}\right|_{-i-3 / 2}$. Using Lemma 5.2 and (6.2), we obtain

$$
\begin{aligned}
\left|\pi_{k} \sigma-\sigma_{k}\right|_{-i-3 / 2} \leqslant C\left(\left|p_{0}\left(G_{h}-G\right) \sigma\right|_{-i-1 / 2}\right. & +\left|p_{0}\left(T_{h}-T\right) f\right|_{-i-1 / 2} \\
& \left.+\left|p_{0} G_{h}\left(\pi_{k} \sigma-\sigma\right)\right|_{-i-1 / 2}\right) .
\end{aligned}
$$

Now if $0<i<n-\frac{1}{2}$, then $p_{0}$ is bounded in $H^{-i-1 / 2}(\partial \Omega)$, and by Lemma 3.1 the first two terms on the right of (6.4) are bounded by $C h^{r+i}\|u\|_{r}$. Finally

$$
\begin{aligned}
\left|G_{h}\left(\pi_{k} \sigma-\sigma\right)\right|_{-i-1 / 2} & <\left|G\left(\pi_{k}-I\right) \sigma\right|_{-i-1 / 2}+\left|\left(G_{h}-G\right)\left(\pi_{k}-I\right) \sigma\right|_{-i-1 / 2} \\
& \leqslant C\left(\left|\left(I-\pi_{k}\right) \sigma\right|_{-i-3 / 2}+h^{i+3 / 2}\left|\left(I-\pi_{k}\right) \sigma\right|\right) \\
& \leqslant C k^{i+3 / 2+i}|\sigma|_{r}
\end{aligned}
$$

where we have again used Lemmas 3.1 and 2.2 , (4.3) and $h<\varepsilon k$. This completes the proof for $0 \leqslant i<n-\frac{1}{2}$. In case $n-\frac{1}{2}<i<r-2$, we cannot infer that $p_{0}$ is bounded in $H^{-(i+1 / 2)}(\partial \Omega)$. Hence we use the triangle inequality in (6.4) and still have to bound $\left|\left(I-p_{0}\right)\left(G_{h}-G\right) \sigma\right|_{-i-1 / 2},\left|\left(I-p_{0}\right)\left(T_{h}-T\right) f\right|_{-i-1 / 2}$ and $\left|\left(I-p_{0}\right) G_{h}\left(\pi_{k} \sigma-\sigma\right)\right|_{-i-1 / 2}$. The result now follows from Lemmas 4.1 and 3.1 , the triangle inequality, (2.7) and (4.3). 
Now, looking at the proof of Theorem 2 from (6.3) on, we see that we have proved the following

THEOREM 3. Under the same hypothesis as in Theorem 2

$\left|\sigma-\sigma_{k}\right|_{-i-3 / 2}$

$$
\leqslant\left\{\begin{array}{l}
C\left(h^{r+i}\|u\|_{r}+k^{\dot{r}+3 / 2+i}|\sigma|_{\dot{r}}\right), \quad 0 \leqslant i \leqslant n-\frac{1}{2}, \\
C\left((k / h)^{i+1 / 2-n} h^{r+i}\|u\|_{r}+k^{\dot{r}+3 / 2+i}|\sigma|_{r}\right), \quad n-\frac{1}{2}<i<r-2 .
\end{array}\right.
$$

As an immediate corollary we have

COROLLARY. Under the same hypothesis as in Theorem 2

$$
\left|\frac{\partial u}{\partial \nu}-\left(\sigma_{k}-\alpha g\right)\right| \leqslant C\left(k^{-n-1} h^{r+n-1 / 2}\|u\|_{r}+k^{\dot{r}}|\sigma|_{\dot{r}}\right) .
$$

Proof. By the triangle inequality and (4.3)

$$
\begin{aligned}
\left|\frac{\partial u}{\partial \nu}-\left(\sigma_{k}-\alpha g\right)\right| & =\left|\sigma-\sigma_{k}\right| \leqslant\left|\pi_{k} \sigma-\sigma\right|+\left|\pi_{k} \sigma-\sigma_{k}\right| \\
& <\left|\pi_{k} \sigma-\sigma\right|+C k^{-n-1}\left|\pi_{n} \sigma-\sigma\right|_{-n-1}+C k^{-n-1}\left|\sigma-\sigma_{k}\right|_{-n-1} \\
& <C\left(k^{\dot{r}}|\sigma|_{\dot{r}}+k^{-n-1}\left|\sigma-\sigma_{k}\right|_{-n-1}\right) .
\end{aligned}
$$

The result now follows from Theorem 3 .

Let us apply Theorem 1 with $r=\dot{r}=2$ to estimate the flux on $\partial \Omega$. The choice which balances the terms is then $k=h^{2 / 5}$ which leads, as in the proof of the corollary, to

$$
\left|\frac{\partial u}{\partial \nu}-\left(\sigma_{k}-\alpha g\right)\right|<C h^{4 / 5}\left(\|u\|_{2}+|\sigma|_{2}\right) .
$$

Applying the Corollary in this case with $n=\frac{1}{2}-\delta$ (discontinuous piecewise polynomials), we obtain

$$
\left|\frac{\partial u}{\partial \nu}-\left(\sigma_{k}-\alpha g\right)\right| \leqslant C h^{8 / 7-\tilde{\delta}}\left(\|u\|_{2}+|\sigma|_{2}\right),
$$

where $\tilde{\delta}=12 \delta[7-2 \delta]^{-1} / 7$. In case $n=\frac{1}{2}$ (e.g. $\dot{S}_{k}$ consists of continuous functions), we obtain

$$
\left|\frac{\partial u}{\partial \nu}-\left(\sigma_{k}-\alpha g\right)\right|<C h^{8 / 7}\left(\|u\|_{2}+|\sigma|_{2}\right) \text {. }
$$

Thus Theorem 2 indicates a different balance between $h$ and $k$ from that suggested by Theorem 1, and a higher rate of convergence in the lower norms and for the flux is proved.

Finally, we see from (6.5) that in case $u$ is very smooth we could obtain the result that for $r$ fixed and any $\delta>0$ we may choose $\dot{r}, n$ and $k$ (as a power of $h$ ) such that

$$
\left|\frac{\partial u}{\partial \nu}-\left(\sigma_{k}-\alpha g\right)\right|=O\left(h^{2 r-2-\delta}\right) .
$$

As an example of Theorem 2 we specialize to the case $i=0, n>\frac{1}{2}$. Then we have

$$
\left\|u-u_{h k}\right\| \leqslant C\left(h^{r}\|u\|_{r}+k^{\dot{r}+3 / 2}|\sigma|_{\dot{r}}\right)
$$


Since $h<\varepsilon k$, the condition $r<\dot{r}+\frac{3}{2}$ is natural. For instance, if $\dot{r}=2$ and $r=2$, then it would be natural to take $h=k^{7 / 4}$. Hence for $k$ sufficiently small the condition $h \leqslant \varepsilon k$ will be satisfied.

7. Computation of $\sigma_{k}$. Let us consider the following discrete surface Laplacian. Define $l_{k}: \dot{S}_{k} \rightarrow \dot{S}_{k}$, with $\dot{S}_{k} \subset H^{1}(\partial \Omega)$, by

$$
\left\langle l_{k} \phi, \theta\right\rangle=\langle\phi, \theta\rangle_{1},
$$

where $\langle\cdot, \cdot\rangle_{1}$ is the inner product in $H^{1}(\partial \Omega)$. (E.g. for $d=2$ we may take $\langle\phi, \theta\rangle_{1}=\langle\phi, \theta\rangle+\left\langle\phi^{\prime}, \theta^{\prime}\right\rangle$ where $\phi^{\prime}$ is the derivative of $\phi$ with respect to arc length along $\partial \Omega$.) Note that the computation of $l_{k} \phi$ involves only the "inversion" of the Gram matrix which in the case of usual finite element spaces will be wellconditioned and sparse. Hence $l_{k} \phi$ is always a relatively inexpensive computation. Now $l_{k}$ is positive definite and symmetric, and we may define $l_{k}^{s}$ in the usual way by taking powers of its eigenvalues. We have the following

LEMMA 7.1. Let $\dot{S}_{k} \subset H^{1}(\partial \Omega)$. Then for $|s| \leqslant 1$ there are constants $C_{0}$ and $C_{1}$ such that for $\phi \in \dot{S}_{k}$

$$
C_{0}|\phi|_{s} \leqslant\left|l_{k}^{s / 2} \phi\right|<C_{1}|\phi|_{s}
$$

Proof. For $s=1$ it is obvious by definition. For $s=-1$ we have that

$$
\begin{aligned}
|\phi|_{-1} & =\sup _{\psi \in H^{1}(\partial \Omega)} \frac{\langle\phi, \psi\rangle}{|\psi|_{1}}=\sup _{\psi \in H^{1}(\partial \Omega)} \frac{\left\langle l_{k}^{-1 / 2} \phi, l_{k}^{1 / 2} p_{0} \psi\right\rangle}{|\psi|_{1}} \\
& \leqslant\left|l_{k}^{-1 / 2} \phi\right| \sup _{\psi \in H^{1}(\partial \Omega)} \frac{\left|l_{k}^{1 / 2} p_{0} \psi\right|}{|\psi|_{1}}=\left|l_{k}^{-1 / 2} \phi\right| \sup _{\psi \in H^{1}(\partial \Omega)} \frac{\left|p_{0} \psi\right|_{1}}{|\psi|_{1}} .
\end{aligned}
$$

Applying Lemma 4.2, we obtain $|\phi|_{-1} \leqslant C\left|l_{k}^{-1 / 2} \phi\right|$. Now

$$
\left|l_{k}^{-1 / 2} \phi\right|^{2}=\left\langle l_{k}^{-1} \phi, \phi\right\rangle \leqslant\left|l_{k}^{-1} \phi\right|_{1}|\phi|_{-1}=\left|l_{k}^{-1 / 2} \phi\right||\phi|_{-1}
$$

by the definition of $l_{k}$. Hence $\left|l_{k}^{-1 / 2} \phi\right| \leqslant|\phi|_{-1}$, and (7.1) is proved for $s=-1$. By interpolation it holds for $-1 \leqslant s \leqslant 1$.

As an application, we combine Lemmas 5.1 and 7.1 to obtain

$$
C_{0}\left|l_{k}^{-1 / 4} \phi\right|^{2} \leqslant\left\langle G_{h} \phi, \phi\right\rangle\left\langle C_{1}\left|l_{k}^{-1 / 4} \phi\right|^{2}\right.
$$

or taking $l_{k}^{1 / 4} \theta=\phi$

$$
C_{0}|\theta|^{2}\left\langle\left\langle\left(l_{k}^{1 / 4}\left(p_{0} G_{h}\right) l_{k}^{1 / 4}\right) \theta, \theta\right\rangle \leqslant C_{1}|\theta|^{2}\right.
$$

for $\theta \in \dot{S}_{k}$. This inequality means that the system with matrix induced by $l_{k}^{1 / 4}\left(p_{0} G_{h}\right) l_{k}^{1 / 4}$ has bounded condition number, and hence we can obtain a solution $\phi$ to the equation

$$
l_{k}^{1 / 4}\left(p_{0} G_{h}\right) l_{k}^{1 / 4} \phi=\psi
$$

to within an accuracy $h^{r}$ by the conjugate gradient method (or some other method) in $O(\ln 1 / h)$ iterations. To apply such a method we need to be able to compute $l_{k}^{1 / 2}\left(p_{0} G_{h}\right)$ for any $\phi \in \dot{S}_{k}$ (cf. [1]). An example of where this is possible is that of smooth splines on a uniform mesh of size $k(d=2)$. In this case we may easily take the discrete Fourier transform for $\phi \in \dot{S}_{k}$. Hence in that case $l_{k}^{1 / 2}$ is easy to compute; in fact using a fast Fourier transform algorithm (cf. [6]) the number of operations required is of the order $k^{-1} \ln k^{-1}$. 
In cases where fractional powers of $l_{k}$ are not easy to compute (e.g. for $d=3$ ) we may apply Lemmas 7.1 and 5.2 to obtain a well-conditioned system. We obtain

$$
C_{0}|\phi|^{2} \leqslant\left|l_{k}^{1 / 2} p_{0} G_{h} \phi\right|^{2}<C_{1}|\phi|^{2}
$$

or

$$
C_{0}|\phi|^{2}\left\langle\left\langle\left(p_{0} G_{h}\right) l_{k}\left(p_{0} G_{h}\right) \phi, \phi\right\rangle \leqslant C_{1}|\phi|^{2}\right. \text {. }
$$

Hence the system induced by $\left(p_{0} G_{h}\right) l_{k}\left(p_{0} G_{h}\right)$ is well-conditioned, and we can use the resulting matrix as a basis for our iteration and apply the conjugate gradient method [1] to obtain a solution to our equations in $O(\ln 1 / h)$ iterations. The solution $\sigma_{k}$ satisfies

$$
p_{0} G_{h} \sigma_{k}=p_{0}\left[g-T_{h} f\right] .
$$

Instead we should consider the well-conditioned system

$$
p_{0} G_{h} l_{k} p_{0} G_{h} \sigma_{k}=p_{0} G_{h} l_{k} p_{0}\left[g-T_{h} f\right] \text {. }
$$

The calculation of $p_{0}$ and $l_{k}$ are inexpensive, and $G_{h}$ is the natural boundary condition solution operator.

The author wishes to thank Professor Lars B. Wahlbin for several suggestions which led to a considerable improvement in this work.

Department of Mathematics

Cornell University

Ithaca, New York 14853

1. O. AxELSSON, "Solution of linear systems of equations: Iterative methods," Sparce Matrix Techniques (V. A. Barker, Ed.), Lecture Notes in Math., vol. 572, Springer-Verlag, Berlin and New York, 1977.

2. I. BABušKa \& A. K. AzIz, "Survey lectures on the mathematical foundations of the finite element method," The Mathematical Foundations of the Finite Element Method with Applications to Partial Differential Equations (A. K. Aziz, Ed.), Academic Press, New York, 1972.

3. I. BABUŠKA, "The finite element method with Lagrangian multipliers," Numer. Math., v. 20, 1973, pp. 179-192.

4. J. H. BRAMble \& J. E. OSBORN, "Rate of convergence estimates for nonselfadjoint eigenvalue approximations," Math. Comp., v. 27, 1973, pp. 525-549.

5. J. H. BRAMble \& R. ScotT, "Simultaneous approximation in scales of Banach spaces," Math. Comp., v. 32, 1978, pp. 947-954.

6. J. W. Cooley \& J. W. TUkey, “An algorithm for the calculation of complex Fourier series," Math. Comp., v. 19, 1965, pp. 297-301.

7. R. S. FALK, “A Ritz method based on a complementary variational principle," R.A.I.R.O. Anal. Numér., v. 10, 1976, pp. 39-48.

8. J. L. Lions \& E. MAgenes, Non-Homogeneous Boundary Value Problems and Applications, Vol. 1, Springer-Verlag, Berlin and New York, 1972.

9. M. SCHECHTER, “On $\mathcal{L}_{p}$ estimates and regularity. II,” Math. Scand., v. 13, 1963, pp. 47-69. 